\title{
The COVID-19 pandemic will redefine the future delivery of glaucoma care
}

\author{
Hari Jayaram $\mathbb{D}^{1,2,3} \cdot$ Nicholas G. Strouthidis ${ }^{1,2,3} \cdot$ Gus Gazzard $^{1,2,3}$
}

Received: 26 April 2020 / Revised: 30 April 2020 / Accepted: 1 May 2020 / Published online: 13 May 2020

(C) The Royal College of Ophthalmologists 2020

Glaucoma is the leading cause of preventable vison loss in the United Kingdom and is responsible for almost one third of new vision impairment certifications [1]. The provision of safe and effective glaucoma care even prior to the current COVID-19 pandemic has been challenging. The British Ophthalmological Surveillance Unit found that up to 22 people per month suffered permanent and severe vision loss as a consequence of delayed follow-up, due to insufficient capacity within hospital eye services [2]. Formal Investigation by the Healthcare Safety Investigation Branch [3] made several recommendations including the need for appropriate specialist-led clinical decision making, clinical pathway redesign, improved referral refinement schemes and validation of risk stratification. Limited progress has been made in recent years despite the issuance of national guidance regarding the conduct of "virtual" glaucoma clinics to help increase capacity [4] and an evidence-based appraisal of current referral refinement strategies [5]. Significant barriers to widespread implementation still exist including an incongruence between organisational structures and emerging models of care and the impact of new roles and relationships upon the function of multiprofessional teams [6].

The COVID-19 pandemic has altered life for the entire population of the United Kingdom in a manner not previously experienced during the lifetime of the majority. Rapid changes to how people live, work and access healthcare have been enforced with limited resistance by citizens. Existing approaches to the monitoring of chronic conditions such as glaucoma, which are not often

Hari Jayaram

h.jayaram@nhs.net

1 Glaucoma Service, Moorfields Eye Hospital NHS Foundation Trust, London, UK

2 UCL Institute of Ophthalmology, London, UK

3 NIHR Moorfields Biomedical Research Centre, London, UK immediately life or sight threatening, have been abandoned because of the priority to preserve life. Almost 120,000 patients attended outpatient consultations within the glaucoma service across the Moorfields Network over the past year with a median age of 68 and almost half aged over 70 . In anticipation of the mitigation strategies implemented by the U.K. government, we have deferred almost 30,000 glaucoma outpatient attendances.

The immediate priority was the task of balancing individual patient's risk of developing glaucoma-related visual impairment during mitigation against their risk of death from COVID-19. The latter was more straightforward to define due to clear government guidance [7], however the former was more challenging. There is currently no nationally agreed, evidence-based risk stratification model for glaucoma. Some have used simple measures of disease staging such as mean deviation as surrogates for risk, but more refined, individualised risk stratification must take into consideration factors related to both glaucoma-related visual impairment and quality of life, which is influenced by the extent of either unilateral or bilateral disease $[8,9]$. Potential decision-making tools also need to incorporate ocular and systemic co-morbidities, rate of disease progression, visual needs and driving status. Further refinement and validation of risk stratification tools is an immediate priority that is essential to safeguard the vision-related quality of life of glaucoma patients across the country.

A "three tier" approach of consultant-led care has been established as the new model of stratified care delivery within the glaucoma service at Moorfields, including consultant-led face-to face clinics, optometrist-led clinics and technician-led "virtual" clinics for those patients at lowest risk [10]. Remote decision making using data collected from glaucoma patients has been shown to be both safe and efficient [11]. Despite this innovation, eighty-six percent of all glaucoma attendances across the Moorfields Network prior to the COVID-19 pandemic still involved a face-to-face visit with either a consultant or specialist 
optometrist. Our response to the current national situation has been to triage patients based upon their COVID-19 and medium-term glaucoma risk, with only clinically urgent cases currently being seen in a hospital setting. The ability to very rapidly risk stratify such large numbers of patients was only possible due to readily accessible data from electronic medical records.

Social distancing measures will become a part of daily life for the foreseeable future. These measures will have direct impact on both the ability and desire of patients to travel to an appointment in a hospital eye clinic. The mean distance from home to local clinic is currently nine miles for glaucoma patients at Moorfields, and concerns over the use of public transport will negatively impact attendances. Clinic capacities will also be reduced by the need to incorporate social distancing within waiting areas. The only viable option to handle the newly exacerbated mismatch between capacity and demand that we now face is to increase the provision of technician-led remote monitoring clinics to facilitate a major shift to consultant-led virtual review. Previously, patients expressed concern at the lack of an interaction with a specialist in "virtual clinics". However, an unintended consequence of the COVID-19 pandemic is that patient perceptions have now changed due to concerns about the risk associated with travel and attendance in a hospital environment.

Collaborations between commissioners, primary care settings and high street optometry providers must be accelerated in order to facilitate the capture of patient data closer to home, away from the hospital environment, but with the capability for all necessary data to be available for consultant-led decision making. A coherent digital strategy to integrate clinical information with visual fields and raw imaging data (to allow automated progression analysis remotely) will be essential. Artificial Intelligence strategies will help streamline care pathways in the future [12], but are not required for the immediate shift to high volume remote review.

Online video consultations have recently been a useful adjunct to emergency eye care, with over 100 urgent consultations a day at Moorfields, but they currently have a limited role in glaucoma care due to the need for ancillary data to make clinical decisions. Recent advances in technologies to provide home monitoring of intraocular pressures and visual fields may enable the expansion of this approach in the future, but as yet remain insufficiently validated for introduction $[13,14]$. Over the recent weeks, many patient concerns have been readily resolved via telephone interaction with a glaucoma consultant, which will prove a useful adjunct to "virtual clinics" in the future. The U.K Ophthalmology Alliance's "Patient Standards for Glaucoma", developed with both patients and clinicians, is a useful resource to optimise the patient experience of remote consultations [15].

The COVID-19 pandemic has completely redefined the landscape in which glaucoma care must now be delivered. New risk stratification models must now account for both glaucoma-related risk of lifetime vision loss and the uncertain risk of harm from COVID-19 infection arising from hospital-based eye-care. These enforced changes also mean we have a once in a generation chance to transform what we do, to focus on those in real need and protect those at risk. Let us hope we are up to the task.

Acknowledgements HJ and NS are employed by Moorfields Eye Hospital NHS Foundation Trust. HJ is supported by the Moorfields Eye Charity. GG is employed by UCL and supported by grants from the National Institute for Health Research (HTA 09/104/40), Moorfields Eye Charity, British Council to Prevent Blindness, Fight For Sight and the International Glaucoma Association. All authors are grateful to the support of the National Institute for Health Research Biomedical Research Centre for Ophthalmology at Moorfields Eye Hospital and the UCL Institute of Ophthalmology. The views expressed in this paper are those of the authors and not necessarily those of any funding body or the Department of Health.

\section{Compliance with ethical standards}

Conflict of interest The authors declare that they have no conflict of interest.

Publisher's note Springer Nature remains neutral with regard to jurisdictional claims in published maps and institutional affiliations.

\section{References}

1. Rahman F, Zekite A, Bunce C, Jayaram H, Flanagan D. Recent trends in vision impairment certifications in England and Wales. Eye. 2020. https://doi.org/10.1038/s41433-020-0864-6. [Epub ahead of print].

2. Foot B, MacEwen C. Surveillance of sight loss due to delay in ophthalmic treatment or review: frequency, cause and outcome. Eye. 2017;31:771-5.

3. Lack of Timely Monitoring of Patients with Glaucoma. Healthcare Safety Investigation 2019/001. United Kingdom: Healthcare Safety Investigation Branch; 2020.

4. Standards for Virtual Clinics in Glaucoma Care in the NHS Hospital Eye Service. Ophthalmic Services Guidance: Royal College of Ophthalmologists; 2016.

5. Glaucoma: diagnosis and management Clinical Guidelines. London: National Institute for Health and Care Excellence; 2017.

6. Turner S, Vasilakis C, Utley M, Foster P, Kotecha A, Fulop NJ. Analysing barriers to service improvement using a multi-level theory of innovation: the case of glaucoma outpatient clinics. Socio Health Illn. 2018;40:654-69.

7. Who's at higher risk from coronavirus. UK National Health Service. 2020. https://www.nhs.uk/conditions/coronavirus-covid-19/ people-at-higher-risk-from-coronavirus/whos-at-higher-risk-fromcoronavirus/. Accessed 25 Apr 2020.

8. Peters D, Heijl A, Brenner L, Bengtsson B. Visual impairment and vision-related quality of life in the Early Manifest Glaucoma 
Trial after 20 years of follow-up. Acta Ophthalmol. 2015; 93:745-52.

9. Ramulu P. Glaucoma and disability: which tasks are affected, and at what stage of disease? Curr Opin Ophthalmol. 2009;20:92-8.

10. Kotecha A, Brookes J, Foster PJ. A technician-delivered 'virtual clinic' for triaging low-risk glaucoma referrals. Eye. 2017;31: 899-905.

11. Clarke J, Puertas R, Kotecha A, Foster PJ, Barton K. Virtual clinics in glaucoma care: face-to-face versus remote decisionmaking. Br J Ophthalmol. 2017;101:892-5.
12. Devalla SK, Liang Z, Pham TH, Boote C, Strouthidis NG, Thiery $\mathrm{AH}$, et al. Glaucoma management in the era of artificial intelligence. Br J Ophthalmol. 2020;104:301-11.

13. Che Hamzah J, Daka Q, Azuara-Blanco A. Home monitoring for glaucoma. Eye. 2020;34:155-60.

14. Holekamp NM. Moving from clinic to home: what the future holds for ophthalmic telemedicine. Am J Ophthalmol. 2018;187: xxviii-xxxv.

15. Patient Standards for Glaucoma: UK Ophthalmology Alliance; 2019. 